ultimate aim, however, will not be achieved in the near future. As Ramón y Cajal once remarked, the structure of the cerebral grey matter is so in- tricate that it defies and will continue to defy for many decades the obstinate curiosity of neurobiologists.

\title{
Anti-anxiety receptors in the brain?
}

\section{from Leslie Iversen}

LIGAND binding techniques are well established as important tools for studies of a variety of biological receptors for peptide hormones and neurotransmitter substances. In most cases such assays have been developed to investigate receptors for which the naturally occurring ligand is already known, for example, insulin, acetylcholine or noradrenaline. In the case of the opiate receptor, however, the discovery of the high affinity binding of radioactively labelled morphine-like drugs to membranes in brain homogenates (Pert \& Snyder Science, 179, 1011 ; 1973) was of a different character, since it allowed description of the precise characteristics and distribution of a receptor mechanism for which no naturally occurring ligand was then known, and it offered a powerful tool for the subsequent discovery of the enkephalins and endorphins as a new class of naturally occurring agonists for a receptor mechanism hitherto described only in terms of the organism's response to opiate drugs.

Such drugs, however, represent only one of several important classes of centrally acting drugs whose pharmacological mechanisms remain obscure. Squires and Braestrup (page 732 of this issue of Nature) now describe their preliminary results in applying a similar rationale to studies of the receptor mechanisms possibly involved in mediating the actions of minor tranquillisers of the benzodiazepine class. These drugs, exemplified by the anti-anxiety agent diazepam (Valium) and the hypnotic drug nitrazepam (Mogadon), have had an enormous commercial success since the discovery of their remarkable psychopharmacological properties in the 1950s. They, and many related analogues in the benzodiazepine series, are able to tame otherwise aggressive laboratory animals, they exhibit CNS muscle relaxant properties, and in man the drugs are used as anticonvulsants and more importantly as anti-anxiety agents. At higher doses they are also effective hypnotics, and since they are much less toxic than barbiturates, have taken an increasing share of the large market for those suffering from anxiety and/or insomnia. Benzodiazepines, needless to say, have been consumed by many millions of people in the industralised nations of the world in which such complaints are endemic.

Despite their unique pharmacological properties and widespread medical use, the mode of action of the benzodiazepines remains unknown. There have been various attempts to explain their effects in terms of current neuropharmacological fashion, that is, by action on CNS catecholamine or indolamine transmitters, but these have been largely unconvincing. Young et al. (Proc. natn. Acad. Sci. U.S.A. 71, 2246 ; 1974) found that the potencies of a series of benzodiazepines in displacing radioactively labelled strychnine from binding sites associated with glycine receptors in mammalian spinal cord correlated fairly well with the potencies of the same drugs in various animal behaviour tests predictive of anti-anxiety properties, and these authors proposed the hypothesis that the drugs might interact with receptors for this inhibitory amino acid transmitter in CNS. However, relatively high concentrations of the drugs were needed to show this effect and subsequent neurophysiological experiments failed to lend support to this hypothesis (Curtis et al. Brit. J. Pharmac. 56, 307; 1976). A more recent proposal has been that the benzodiazepines might in some way mimic or enhance the effects of another inhibitory amino acid transmitter, $\gamma$-aminobutyric acid (GABA) at CNS receptor sites. This hypothesis was based on both biochemical and neurophysiological evidence (Polc et al. N. S. Archiv. Pharmac. 284, 319; 1974; Costa et al. Adv. Biochem. Psychopharmac. 14, 113; 1975), and has attracted a wide following. Nevertheless, it has proved difficult to demonstrate any direct interaction between benzodiazepines and GABA receptors, although recent neurophysiological evidence has shown that these drugs are able to exert inhibitory effects on the firing of single cells in rat brain similar to those seen on application of GABA (Dray \& Straughan J. Pharm. Pharmac. 28, 314 ; 1976).

Squires and Braestrup found that high specific activity ${ }^{3} \mathrm{H}$-diazepam is bound to saturable sites in rat brain membrane preparations with very high affinity--half saturation being achieved at a drug concentration of only $2.6 \mathrm{nM}$. When low concentrations of the labelled drug were used more than $90 \%$ of the total binding was attributable to this single class of high affinity sites. More importantly, they showed that the potencies of 21 different nonradioactive benzodiazepine drugs in competing with labelled diazepam for binding showed a highly significant correlation with the known pharmacological potencies of these drugs determined previously in various animal tests known to be predictive for antianxiety properties in man. The potencies of the drugs tested in both the in vivo and in vitro tests ranged over four orders of magnitude. On the other hand, none of the known amine or amino acid transmitters (including glycine and GABA), or any of the drugs known to interact with their receptors, had any inhibitory effects on ${ }^{3} \mathrm{H}$-diazepam binding. Other minor tranquillisers, such as meprobamate, barbiturates or ethanol, were also ineffective as inhibitors, as were opiate drugs.

The authors therefore, claim to have described a saturable high affinity binding mechanism whose pharmacological specificity suggests that it may have some important relation to the mode of action of the benzodiazepines in whole animal experiments or in man. It is also interesting to note that the highest density of ${ }^{3} \mathrm{H}$-diazepam binding sites was found in the frontal and occipital cortex of rat brain, areas not known to be enriched in any of the known transmitter substances.

If their hypothesis is correct, and caution is certainly still needed in extrapolating from these preliminary biochemical results, their findings may have a major impact on our future understanding of the mode of action of this class of psychotropic drugs. By analogy with the discovery of the opiate receptor, the benzodiazepine receptor assay may even offer a simple means for the discovery of the naturally occurring ligand for such receptorsthe brain's own anti-anxiety substance?

Further developments in this field will certainly be awaited-anxiously.

\section{A hundred years ago}

AT the last meeting of the French Anthropological Society, a long report was read which showed that Druidism was not quite extinct in Britanny, some country people still adhering to Pagan practices in spite of the priests' exertions. It was noticed that the clergy were anxious to destroy menhirs and other similar relics. A petition has been sent to the ministry to put a stop to this iconoclastic zeal.

From Nature, 1519 April, 539; 1877. 